\title{
ATP Luminescence-Based Motility-Invasion Assay
}

BioTechniques 33:98-106 (July 2002)

\author{
Suzanne M. de la Monte, \\ Stephanie A. Lahousse, Jade \\ Carter, and Jack R. Wands \\ Brown Medical School and \\ Rhode Island Hospital, \\ Providence, RI, USA
}

\section{INTRODUCTION}

Directional migration assays largely rely on the use of Boyden chambers or Transwell ${ }^{\mathrm{TM}}$ culture inserts (Corning Costar, Acton, MA, USA) in which porous polycarbonate or polypropylene membranes separate the seeded cells from the chemotactic factor in the medium below. Motility assays are performed by seeding cells into the upper portion of the chamber and then allow ing cells to migrate for time intervals ranging from several hours to several days. The principal objective is to determine the proportion of cells that migrate through the pores and either remain attached to the undersurface of the mem brane or fall to the bottom of the well. Invasion assays are similarly constructed, except the membranes are coated with extracellular matrix molecules such as laminin, fibronectin, collagen type IV, or tenascin $(1,2,7)$, complex matrices such as Matrigel ${ }^{\mathrm{TM}}$ (BD Biosciences Clontech, Palo Alto, CA, USA) $(11,13,18)$, or monolayers of cells $(21)$.

Both colorimetric- and fluorimetricbased assays are widely used to quantify cell migration. For the colorimetricbased assays, the cells can be labeled with 3-(4,5-dimethylthiazol-2-yl)-2,5diphenol tetrazolium bromide (MTT) and, after removing the nonmigrated cells from the upper surface of the membrane with a cotton swab, the cells that have migrated through the pores and remain attached to the undersurface of the membrane or that fall to the bottom of the well are solubilized to measure the formazen reaction product (13). Alternatively, the migrated cells can be stained with colorimetric dyes such as Toluidine blue and counted using microscopy $(9,11)$. However, these assays can produce inconsistent results because of poor cell adhesion to the bottom of the wells and variability in staining intensity and background generated by the protein coating on the membrane (19). Fluorescence-based assays offer a major improvement over colorimetric motility assays because of increased sensitivity and more objective methods of quantifying cell density using detectors such as fluorescence microplate readers. Several approaches have been successfully employed, including fluorescent cell tagging with compounds such as Calcein AM (Molecular Probes, Eugene, OR, USA), lipophilic fluorescent dyes (which exhibit excellent uptake and long-term retention) $(5,14,20)$, or by transfection of GFP expression vectors. A major draw back to these approaches is the inability to quantify the entire population of cells in each assay.

More recently, better-designed assays such as the fluorescence-assisted transmigration invasion and motility assay (FATIMA ${ }^{\mathrm{TM}}$; Tecan, Durham, NC, USA) have been designed to accomplish this goal (19). The FATIMA plates incorporate a specific porous membrane that is designed to shield fluorescent light in the wavelength range of 450-550 nm and is based on total cell labeling with lipophilic carbocyanine dyes (Molecular Probes). The lipophilic fluorescent dyes enable the long-term labeling required to study migration over a period of days $(6,10,17)$. However, because a specialized microplate fluorometer (FluoroCount; Perkin Elmer Life Science, 
Gaithersburg, MD, USA) with independent top and bottom measurement capabilities and a FATIMA computer interface (Tecan) are required to quantify and interpret the results, this method of measuring cell migration and invasiveness may be too expensive and time consuming for most investigators. We have developed a luminescence-based assay that also quantifies nonmigrated, migrated-adherent (transmigrated), and migrated nonadherent cells in each assay chamber. The assay is simple, quantitative, and performed using a luminescence microplate reader.

\section{MATERIALS AND METHODS}

\section{Directional Motility Assay}

Directional motility was measured using blind well chambers partitioned with $13 \mathrm{~mm}$ diameter, $8 \mu \mathrm{M}$ pore polycarbonate membranes (Neuro Probe, Gaithersburg, MD, USA). The studies described utilized rat 9L glial cells, but other cell lines including hepatocellular carcinoma, cholangiocarcinoma, neuroblastoma, and 293 cells were successfully evaluated. Trypsinized cells were washed three times in 10 volumes of serum-free DMEM containing $2 \mathrm{mM}$ glutamine and then resuspended in serum-free medium at a density of $10^{6}$ cells $/ \mathrm{mL}$. In the lower chamber, $200 \mu \mathrm{L}$ medium supplemented with $1 \%$ heatinactivated FCS, insulin (10 nM), or insulin-like growth factor type 1 (IGF-1; $5 \mathrm{ng} / \mathrm{mL}$ ) were supplied as the chemoattractant. After assembly, $10^{5}$ viable cells (Trypan blue exclusion) suspended in $100 \mu \mathrm{L}$ serum-free medium were added to the chamber. The chambers were placed in a $\mathrm{CO}_{2}$-humidified incubator, and migration was allowed to proceed for $4 \mathrm{~h}$, which was determined to be sufficient to measure cell motility and invasiveness in pilot studies. How ever, the ATP luminescence-based motility-invasion (ALMI) assay was found suitable for measuring motility in overnight or 24-h experiments.

The ATPLite ${ }^{\mathrm{TM}}$ assay (Perkin Elmer Life Science), which quantifies ATP present in viable intact cells, was used to measure nonmigrated, migrated-adherent, and migrated-nonadherent cell populations. The cells remaining inside the chamber on the upper surface of the membrane (nonmigrated) were harvested with a sterile cotton swab by applying gentle pressure and twirling. Briefly, after absorbing the medium, the cotton swab was twirled, applying gentle pressure to remove the cells completely, without puncturing the membrane or pushing it into the lower chamber. The cotton swab was then inserted into a well of a 96-well black plate that contained $100 \mu \mathrm{L}$ distilled water plus $25 \mu \mathrm{L}$ ATP lysis buffer (Perkin Elmer Life Science). The applicator stick was clipped with pruning sheers to enable the cotton swab to fit entirely in the well. To harvest migrated-adherent cells, the blind well cham ber was disassembled and the membrane, devoid of nonmigrated cells, was placed into a second well containing $100 \mu \mathrm{L}$ distilled water plus $25 \mu \mathrm{L}$ ATP lysis buffer. The migrated-nonadherent cells were harvested by resuspending the cells in the lower compartment of the blind well, transferring them to an Eppendorf ${ }^{\circledR}$ tube, pelleting the cells in a microcentrifuge at $12000 \times g$ for $30 \mathrm{~s}$, and then aspirating the medium. The lysate obtained by adding $100 \mu \mathrm{L}$ water plus $25 \mu \mathrm{L}$ ATPLite lysis buffer to the pellet was placed into a third well of the 96-well plate. After harvesting the cells from all the chambers, the plate was covered with an adhesive sheet and incubated at room temperature for $5 \mathrm{~min}$, with gentle platform agitation to ensure thorough cell lysis.

ATPLite substrate solution was prepared according to the manufacturer's instructions. Briefly, the lyophilized ATPLite substrate was solubilized in 5 $\mathrm{mL}$ ATPLite buffer (provided by the manufacturer), and $25 \mu \mathrm{L}$ substrate were added to each well. The plate was covered and incubated for an additional 2 min with gentle platform agitation. After a 2-min interval of dark adaptation, luminescent counts/s were measured for $5 \mathrm{~s} /$ well in a TopCount microplate reader (Perkin Elmer Life Science). We routinely use black plates in our assays to minimize cross talk among the wells. The data were exported and analyzed using Microsoft ${ }^{\circledR}$ Ex$\mathrm{cel}^{\circledR}$ to calculate the percentages of nonmigrated, migrated-adherent, and migrated-nonadherent cells in each assay chamber. 
Controls were included in all experiments to verify uniform seeding and complete cell harvesting. The com pleteness of cell harvesting was monitored by the microscopic examination of the membranes and blind wells from replicate assays that were not used to measure ATPLite luminescence. No cells remained on either the upper surfaces of the membranes or in the blind wells following the procedure described above. In addition, samples (replicates of 16 or 24) containing $10^{5}$ cells were seeded into the wells of a 96well plate in the presence or absence of a chemoattractant. Half the samples were immediately lysed and assayed for ATPLite luminescence, and the other half were incubated at $37^{\circ} \mathrm{C}$ and assayed for ATPLite luminescence in parallel with the motility assays. Typically, ATPLite counts/s measured in the cells placed directly into the 96-well plates equaled the total ATPLite counts/s measured in the three compartments (nonmotile + motile-adherent + motilenonadherent) of a motility assay chamber, and the difference between the ATPLite luminescence values measured in the cells that were lysed immediately or after $4 \mathrm{~h}$ incubation was generally negligible $(<5 \%)$. Note that we have obtained consistent results $(\mathrm{CV}<$ $10 \%$ ) irrespective of small differences in the initial cell density, perhaps because the data were expressed as percentages of motile cells within an entire population.

\section{Luminescence-Based Invasion Assay}

The luminescence-based invasion assay was constructed as described above, except that the membranes were precoated with extracellular matrix molecules such as gelatin $(0.2 \mathrm{mg} / \mathrm{mL})$, collagen type IV $(0.5 \mathrm{mg} / \mathrm{mL})$, laminin $(17.5 \mu \mathrm{g} / \mathrm{mL})$, extracellular matrix com pound $(1 \mu \mathrm{g} / \mathrm{mL})$, and fibronectin $(0.5$ $\mu \mathrm{g} / \mathrm{mL}$ ). Except for the collagen type IV, all matrix molecules were diluted in TBS (50 mM Tris, $\mathrm{pH} 7.4,150 \mathrm{mM}$ $\mathrm{NaCl}$ ). On the day of use, membranes were coated with fibronectin, laminin, or gelatin for $1 \mathrm{~h}$ at room temperature. Membranes were coated with extracellular matrix compound for $5 \mathrm{~min}$ at $4^{\circ} \mathrm{C}$. Collagen type IV was diluted in $0.25 \%$ acetic acid, and the membranes were coated for $2 \mathrm{~h}$ at $37^{\circ} \mathrm{C}$. All coated mem branes were allowed to air dry for 1-2 h. The cells were then seeded directly onto the coated surfaces, and invasion was allowed to proceed for 4-24 h. Luminescent counts/s were used to determine the percentages of nonmotile/noninvading (counts/s cells from the upper chamber and membrane surface/the counts/s of the cells from all compartments $\times 100$ ), motile/invading-adherent (counts/s of the cells adherent to membrane after removing cells from upper surface/counts/s of cells from all com partments $\times 100$ ), and motile/invadingnonadherent cells (counts/s of the cells in the medium of the lower chamber/ counts/s of the cells from all compartments $\times 100$ ) in each assay. The values for eight or more replicate assays were used to make intergroup statistical com parisons with Student's $t$ tests or ANOVA (significance levels: $P<0.05$ ).

\section{Source of Reagents}

All cell lines were obtained from the ATCC (Manassas, VA, USA). Humulin insulin was purchased from Eli Lilly \& Co. (Indianapolis, IN, USA). Recombinant human IGF-1, all matrix compounds used for invasion assays, and all cell culture reagents and chemicals were purchased from Sigma (St. Louis, MO, USA).

\section{RESULTS}

Rat 9L glial cells were used to measure motility and invasiveness because these cells were found to be highly motile and invasive relative to other cell types; therefore, the effects of inhibitory substances could be readily detected and quantified. To determine the relationship between the cell number and ATPLite luminescence, known numbers of cells suspended in medium containing $1 \%$ FCS were seeded into wells of 96-well black plates using an $\mu L T R O N$ 8-channel programmable automated pipetting device (MLA Systems, Pleasantville, NY, USA) and then immediately assayed for ATP content. ATPLite luminescence counts/s increased linearly with the viable cell number over a broad range (Figure 1A). However, because the slope of the regression line relating the cell density to ATPLite counts/s varies with cell type, it is necessary to characterize the linear dynamic range of this assay for each cell line. To determine if ATP levels were altered by growth factor exposure versus deprivation during the 4-h interval required to measure motility or invasion, the $9 \mathrm{~L}$ glial cells suspended in medium containing $5 \mathrm{ng} / \mathrm{mL}$ IGF-1 or no growth factor were seeded into 96 -well plates and incubated for $4 \mathrm{~h}$ at $37^{\circ} \mathrm{C}$, after which the cells were lysed and ATP was
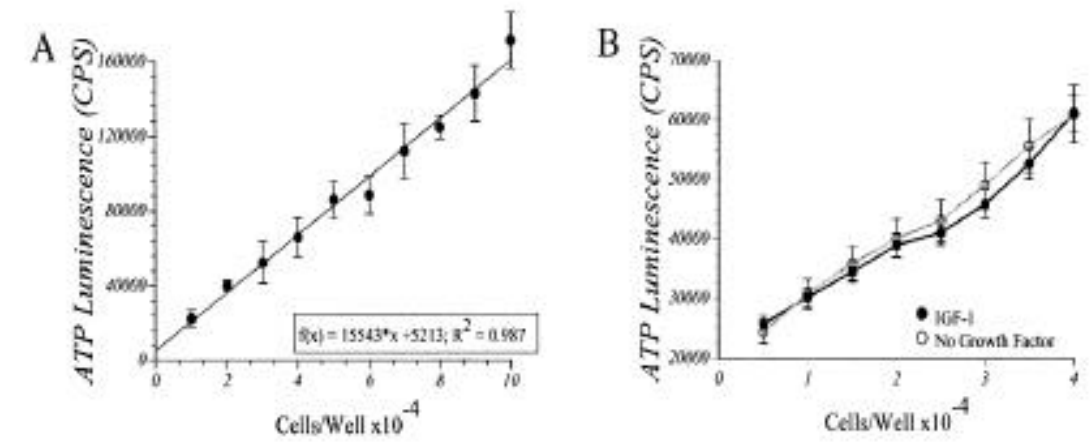

Figure 1. ATP levels are linearly correlated with cell density. (A) Linear relationship between the cell number and ATPLite luminescence. Rat 9L glial cells were seeded into 96-well plates at the densities indicated with medium containing $1 \%$ FCS. The cells were immediately lysed in ATPLite buffer, and ATP levels were detected using a luminescence-based assay (see Materials and Methods). (B) Correlation between the cell density and ATP levels in 9L glial cell cultures seeded with IGF-1 or no growth factor in the medium. The cells were incubated at $37^{\circ} \mathrm{C}$ for $4 \mathrm{~h}$ and then assayed for ATP to simulate conditions used for motility assays. Each point represents the $\overline{\mathrm{x}} \pm$ SD of the ATP luminescence counts/s obtained from eight replicate culture wells. 
measured. Virtually identical levels of ATPLite luminescence were detected, irrespective of growth factor stimulation (Figure 1B). ATPLite luminescence was not detected (counts/s $<50$ ) in cell-free supernatants, cotton swabs, or unused polycarbonate membranes.

We examined the effect of growth factor stimulation on 9L glial cell migration. Parallel cultures employed
IGF-1, insulin, or FCS as the chemoattractant, and the percentages of migrated-adherent and migrated-nonadherent cells were calculated using ATPLite luminescence counts/s. IGF-1 provided the most potent stimulus for $9 \mathrm{~L}$ glial cell migration, while $1 \%$ FCS was least effective (Figure 2, A and B). However, IGF-1-stimulated cells were significantly less prone than insulin- or $1 \%$

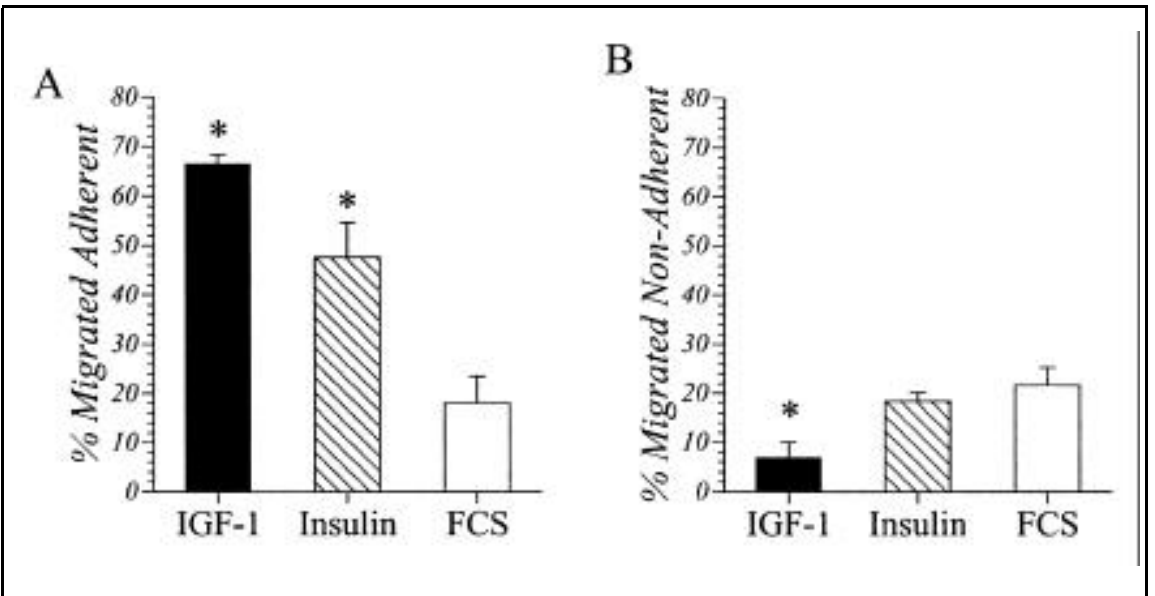

Figure 2. Effects of IGF-1, insulin, and FCS stimulation on 9L glial cell motility. The ATPLite ALMI assay was used to quantify the directional motility of the cells using Neuro Probe blind well chambers equipped with porous $(8 \mu \mathrm{M})$ polycarbonate membranes. The $10^{5}$ cells were seeded into the chambers in serum-free medium, and IGF-1 (5 ng/mL; solid), insulin (10 nM; hatched), or $1 \%$ FCS (speckled) was supplied as the trophic factor in the medium below the membrane. Migration was allowed to proceed for $4 \mathrm{~h}$, after which the cells were harvested from the upper and lower surfaces of the membrane and the bottom of the well. The cell number was determined using ATPLite reagents. The percentages of (A) migrated-adherent (attached to the undersurface of the membrane) and (B) migrated-nonadherent (located at the bottom of the well) cells ( $\overline{\mathrm{x}} \pm$ SD of six replicate assays) are depicted in the graphs $(* P<0.001$ relative to FCS by ANOVA with the Fisher LSD post-hoc test of significance).

A

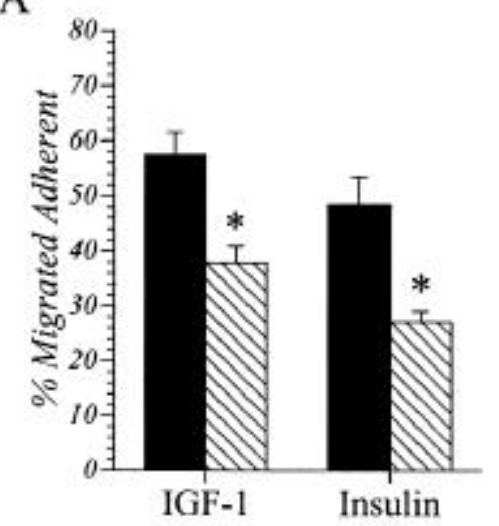

B

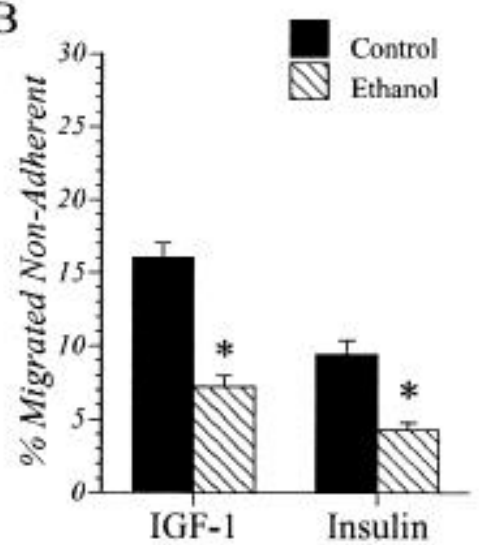

Figure 3. Ethanol inhibition of 9L glial cell migration demonstrated with the ALMI assay. Control and ethanol-treated cells (50 $\mathrm{mM}$ for four days) were seeded into Neuro Probe blind well chambers as described (Figure 2 legend) and stimulated with IGF-1 $(5 \mathrm{ng} / \mathrm{mL})$ or insulin $(10 \mathrm{nM})$. Migration was allowed to proceed for $4 \mathrm{~h}$, after which the cells were harvested, and ATP levels were quantified using ATPLite. The graphs depict the $\bar{x} \pm$ SD percentages of migrated-adherent (A) and migrated-nonadherent (B) cells measured in eight replicate assays ( ${ }^{*} P<0.001$ relative to control by Student's $t$ test).

FCS-stimulated cells to migrate through the membrane and fall to the bottom of the well (Figure 2B).

In a second experiment, we examined the effects of ethanol exposure on insulin- and IGF-1-stimulated 9L glial cell migration. Previous studies demonstrated major inhibitory effects of ethanol on insulin and IGF-1-stimulated functions in central nervous system cells $(4,8,12,15,16,22)$. An important consequence of gestational exposure to ethanol is impaired migration in the central nervous system during development $(3,8)$. Therefore, it was of interest to determine if the ethanol inhibition of growth factor-stimulated cell migration could be detected using the ALMI assay. Subconfluent 9L glial cell cultures were exposed to $50 \mathrm{mM}$ ethanol or nothing for four days and then analyzed for directional motility using IGF-1 or insulin as the chemoattractant. The ethanol-exposed cells manifested significantly reduced insulin and IGF-1stimulated directional motility, but the inhibitory effects of the ethanol were more pronounced in the insulin-stimulated cultures (Figure 3, A and B). Ethanol impaired the motility in nonadherent cells to a greater extent (approximately $50 \%$ ) than in adherent cells (approximately 30\%) (Figure 3). These results could not be explained by ethanol inhibition of cell proliferation or viability because the motility assays were performed within a 4-h period, whereas the inhibitory effects of ethanol on cell growth and survival are detected over 12- or 24-h intervals $(4,12,22)$.

In a third experiment, 9L glial cell invasiveness was measured using mem branes coated with gelatin, laminin, fibronectin, collagen type IV, or extracellular matrix compound cocktail just before use. Relative to the control, uncoated membranes, fibronectin, and laminin coatings were associated with increased transmigration (migrated-adherent population), while the collagen type IV and extracellular matrix com pound coatings resulted in the significantly reduced transmigration of $9 \mathrm{~L}$ glial cells (Figure 4). However, extracellular matrix compound precoating resulted in significantly increased 9L glial cell invasion and passage into the lower chamber (Figure 4B). Therefore, the effects of different extracellular ma- 
trices on cellular invasiveness can be measured using the ALMI assay.

\section{DISCUSSION}

We developed a highly sensitive luminescence-based assay to measure directional migration and invasiveness in vitro. The major advantages of the ALMI assay are (i) all viable cells (including nonmigrated cells) are accounted for, thus providing a true denominator for quantifying motility; (ii) the experimental conditions can be modified easily; (iii) the results are reproducible, quantitative, and can be com pared among different experiments; $(i v)$ the protocol is straightforward, such that 24 assay chambers can be harvested and analyzed within $1 \mathrm{~h}$; and $(v)$ the expenses are minimized by the use of standard equipment available in most laboratories.

A major drawback to colorimetricbased motility and invasion assays is that, to assess cell migration, the nonmigrated cells must be removed from the upper surface of the membrane and therefore cannot be used as the denominator to quantify the percentage of migrated cells. In addition, because cells may die or proliferate during the period of migration, the lack of a denominator further renders colorimetric-based motility assays inaccurate. These problems are circumvented using the ATPLite luminescence-based motilityinvasion assay because viable cells swabbed from the upper membrane surface can be detected easily. Moreover, because cotton swabs do not contain ATP, they do not generate background signals, as was verified in the initial design of the assay. This feature provides an additional advantage over colorimetric-based motility and invasion assays that can be hampered by variable nonspecific staining of the membrane, thus limiting the use of absorbance of eluted dye as an index of cell number.

As constructed, the ALMI assay provides maximum flexibility for experimental conditions. The reusable blind well chambers utilize polycarbonate or polypropylene membranes that are available in a range of pore sizes, can be coated with a variety of extracellular matrix materials or cells to study invasiveness, and can be purchased from independent vendors. The only drawback associated with this system is that the membranes must be individually handled and coated. Although most technical personnel can manage the procedure, $2 \mathrm{~h}$ are required to coat 24 membranes, and another $2 \mathrm{~h}$ are needed to dry the membranes. We did not at-

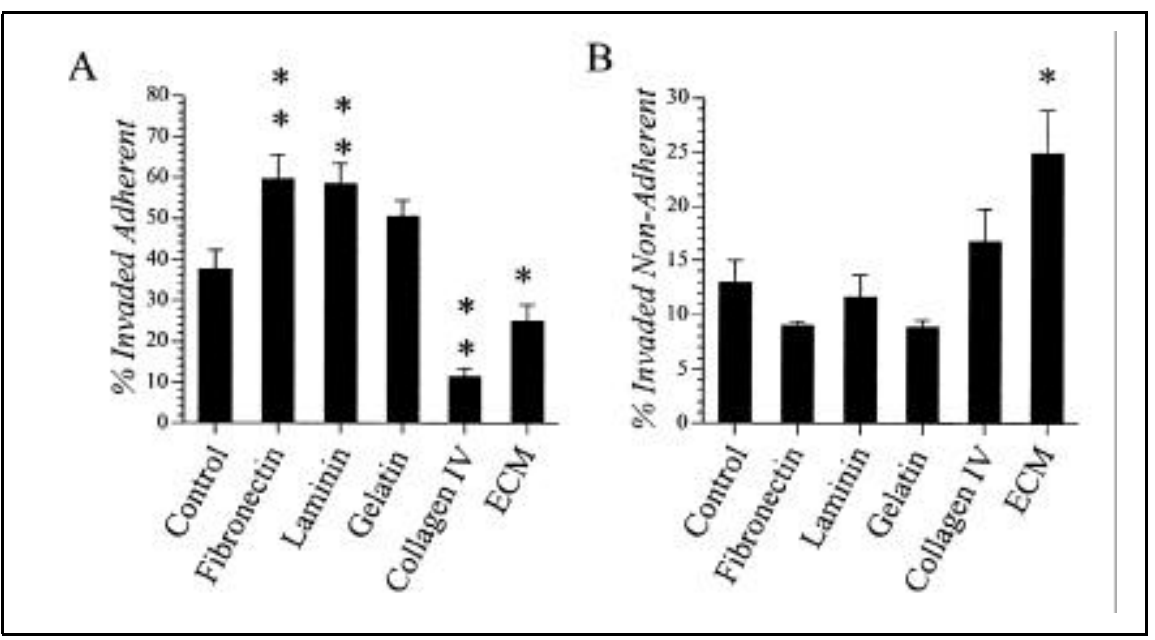

Figure 4. Measurement of 9L glial cell invasiveness using polycarbonate membranes coated with nothing (control), fibronectin $(0.5 \mu \mathrm{g} / \mathrm{mL})$, laminin $(17.5 \mu \mathrm{g} / \mathrm{mL})$, gelatin $(0.2 \mathrm{mg} / \mathrm{mL})$, collagen type IV $(0.5 \mathrm{mg} / \mathrm{mL})$, or extracellular matrix compound $(\mathrm{ECM} ; 1 \mu \mathrm{g} / \mathrm{mL})$. The cells were seeded into Neuro Probe blind well chambers as described (Figure 2 legend) and stimulated with 1\% FCS. Migration/invasion was allowed to proceed for $4 \mathrm{~h}$, after which the cells were harvested to measure ATP levels as described in the text. The graphs depicts the $\bar{x} \pm$ SD percentages of (A) adherent and (B) nonadherent invaded cells in six replicate assays $(* * P<0.001 ; * P<0.05$ relative to the control by ANOVA with the Fisher LSD post-hoc test of significance).

tempt to precoat and store the mem branes for later use; however, this approach must be feasible because precoated membranes provided with Boyden chamber-type cell culture inserts (Transwell) are commercially available. In this regard, it is noteworthy that we have successfully adapted the ALMI assay to Transwell chambers. The procedures for harvesting nonmigrated- and migrated-nonadherent cells are the same as described earlier, except that the migrated-adherent population must also be harvested by swabbing the undersurface. The results obtained using the blind well and Transwell systems were virtually identical, except the blind well assay system is reusable and considerably less expensive.

Because cell motility and invasiveness are calculated as percentages of the total cell number (ATPLite luminescence counts/s in all compartments), the reproducibility of the assay was relatively high and usually associated with a CV of less than $10 \%$. This allows the investigator to compare trends among different experiments and experimental conditions. The ability to generate information about the percentages of migrated-adherent (attached to the undersurface of the membrane) and migrated-nonadherent (cells in the lower chamber) also permits the analysis of two distinct aspects of cell motility or invasiveness. For example, significant differences in the percentages of migrated-nonadherent but not transmigrated (migrated-adherent) populations would suggest that cell adhesion mechanisms should be evaluated.

The protocol as designed is relatively straightforward and easy to replicate. The ease of the assay permits 24 assay chambers to be harvested and analyzed within a $1-\mathrm{h}$ period. The only problems encountered were $(i)$ the application of excessive pressure to harvest the nonmigrated cells with the cotton swab caused the membrane to be pushed through to the lower chamber, yielding artificially high percentages of migrated cells; (ii) the membrane must be completely submerged in lysis solution to accurately measure the migrated-adherent cell population; and (iii) the chambers must be cleaned with a dilute antiseptic solution between assays to prevent the luminescence detection of bacteria. 
In our experience, with regard to the first problem, it is always immediately apparent that a membrane has been accidentally plunged into the blind well. However, the problem can be minimized with practice $(8-10$ chamber harvests), as indicated by the fact that our current error rate with experienced personnel is less than $2 \%$. The inclusion of 12-16 replicate assays, particularly in the initial studies, can be used to establish baseline results for given cell types or experimental conditions. Significant errors in harvesting nonmigrated cells should be suspected if one result differs substantially (>40\%) from those obtained with $80 \%-90 \%$ of the replicate assays. Although conventional motility assays are unaffected by this potential problem, their failure to account for all cell populations prohibits the calculation of the percentages of motile cells that could be used for comparison among different experimental conditions. Visual inspection of the 96well assay plates was sufficient to determine if the membranes were adequately submerged to ensure the complete lysis of the cells adhering to the undersurfaces. To prevent bacterial contamination, the chambers were soaked in $1 \%$ BACDOWN $^{\circledR}$ Detergent Disinfectant (Decon Laboratories, Bryn Mawr, PA, USA) for $30 \mathrm{~min}$ and then thoroughly rinsed in distilled water before each use. Under a laminar flow hood, the chambers were dried with sterile paper wipes and cotton swabs.

Another major advantage of the ALMI assay is that the costs are minimized by the use of equipment available in most laboratories. Although the FATIMA accomplishes the same goals as the ALMI assay, it requires specialized plates and membranes to shield fluorescent light within a specified wavelength range and a specialized microplate fluorometer with a dedicated computer interface. With the aid of a 96well luminescence microplate reader, results from multiple replicate assay chambers and different experimental conditions can be obtained within 1-2 $\mathrm{h}$ after the period of cell migration. In conclusion, the ALMI assay is a simple luminescence-based method of quantifying cell migration and invasiveness. Because all cells are accounted for, intergroup comparisons can be made using the calculated percentages of nonmigrated, migrated-adherent (transmigrated), and migrated-nonadherent cells in each chamber.

\section{ACKNOWLEDGMENTS}

Supported by National Institutes of Health grant nos. CA-35711, AA02666, and AA-02169 to J.R.W., and grant no. AA-11431 to S.M.d.l.M. and J.R.W.

\section{REFERENCES}

1.Chattopadhyay, N., S. Ray, N. Biswas, and A. Chatterjee. 1999. Effect of all-transretinoic acid on integrin receptors of human cervical cancer (SiHa) cells. Gynecol. Oncol. $75: 215-221$

2.Chicoine, M.R. and D.L. Silbergeld. 1997. Mitogens as motogens. J. Neurooncol. 35:249-257.

3.Clarren, S.K., E.J. Alvord, S.M. Sumi, A.P. Streissguth, and D.W. Smith. 1978. Brain malformations related to prenatal exposure to ethanol. J. Pediatr. 92:64-67.

4.de la Monte, S.M., N. Ganju, K. Banerjee, N.V. Brown, T. Luong, and J.R. Wands. 2000. Partial rescue of ethanol-induced neuronal apoptosis by growth factor activation of phosphoinositol-3-kinase. Alcohol Clin. Exp. Res. 24:716-726.

5.Garrido, L., A. Bogdanova, L.L. Cheng, B. Pfleiderer, E. Tokareva, J.L. Ackerman, and T.J. Brady. 1996. Detection of silicone migration and biodegradation with NMR. Curr. Top. Microbiol. Immunol. 210:49-58.

6.Godement, P., J. Vanselow, S. Thanos, and F. Bonhoeffer. 1987. A study in developing visual systems with a new method of staining neurones and their processes in fixed tissue. Development 101:697-713.

7.Iwamoto, Y. and Y. Sugioka. 1992. Use of a reconstituted basement membrane to study the invasiveness of tumor cells. Adv. Exp. Med. Biol. 324:141-149.

8.Miller, M. 1992. Effects of prenatal exposure to ethanol on cell proliferation and neuronal migration, p. 47-69. In M.W. Miller (Ed.), Development of the Central Nervous System: Effects of Alcohol and Opiates. John Wiley \& Sons, New York.

9.Muir, D., L. Sukhu, J. Johnson, M.A. Lahorra, and B.L. Maria. 1993. Quantitative methods for scoring cell migration and invasion in filter-based assays. Anal. Biochem. 215:104-109.

10.Ragnarson, B., L. Bengtsson, and A. Haegerstrand. 1992. Labeling with fluorescent carbocyanine dyes of cultured endothelial and smooth muscle cells by growth in dyecontaining medium. Histochemistry 97:329333.

11.Repesh, L.A. 1989. A new in vitro assay for quantitating tumor cell invasion. Invasion Metastasis 9:192-208.
12.Resnicoff, M., M. Rubini, R. Baserga, and R. Rubin. 1994. Ethanol inhibits insulin-like growth factor-1-mediated signaling and proliferation of C6 rat glioblastoma cells. Lab. Invest. 71:657-662.

13.Sasaki, C.Y. and A. Passaniti. 1998. Identification of anti-invasive but noncytotoxic chemotherapeutic agents using the tetrazolium dye MTT to quantitate viable cells in Matrigel. BioTechniques 24:1038-1043.

14.Schratzberger, P., C.M. Kahler, and C.J. Wiedermann. 1996. Use of fluorochromes in the determination of chemotaxis and haptotaxis of granulocytes by micropore filter assays. Ann. Hematol. 72:23-27.

15.Seiler, A.E., A. Henderson, and R. Rubin. 2000. Ethanol inhibits insulin receptor tyrosine kinase. Alcohol Clin. Exp. Res. 24:18691872.

16.Seiler, A.E., B.N. Ross, J.S. Green, and R. Rubin. 2000. Differential effects of ethanol on insulin-like growth factor-I receptor signaling. Alcohol Clin. Exp. Res. 24:140-148.

17.Serbedzija, G.N., S.E. Fraser, and M. Bronner-Fraser. 1990. Pathways of trunk neural crest cell migration in the mouse embryo as revealed by vital dye labeling. Development 108:605-612.

18.Simon, N., A. Noel, and J.M. Foidart. 1992. Evaluation of in vitro reconstituted basement membrane assay to assess the invasiveness of tumor cells. Invasion Metastasis 12:156-167.

19.Spessotto, P., E. Giacomello, and R. Perris. 2000. Fluorescence assays to study cell adhesion and migration in vitro, p. 321-344. In C. Sreuli and M. Grant (Eds.), Extracellular Matrix Protocols. Humana Press, Totowa, NJ.

20.Sunder-Plassmann, G., R. Hofbauer, G. Sengoelge, and W.H. Horl. 1996. Quantification of leukocyte migration: improvement of a method. Immunol. Invest. 25:49-63.

21.Waller, C.A., M. Braun, and V. Schirrmacher. 1986. Quantitative analysis of cancer invasion in vitro: comparison of two new assays and of tumor sublines with different metastatic capacity. Clin. Exp. Metastasis 4:73-89.

22.Xu, Y.Y., K. Bhavani, J.R. Wands, and S.M. de la Monte. 1995. Ethanol inhibits insulin receptor substrate-1 tyrosine phosphorylation and insulin-stimulated neuronal thread protein gene expression. Biochem. J. 310:125-132.

Received 27 August 2001; accepted 11 February 2002.

Address correspondence to:

Dr. S.M. de la Monte

Pierre Galletti Research Building

Rhode Island Hospital

55 Claverick Street, Room 419

Providence, RI 02903, USA

e-mail: delamonte@hotmail.com

For reprints of this or any other article, contact Reprints@BioTechniques.com 